\title{
La transformation des matières dures d'origine animale en Gaule romaine : ateliers urbains et artisans itinérants
}

Transformation of hard animal material in the Roman period in Gaul: urban workshops and itinerant artisans

\section{Isabelle Rodet-Belarbi}

\section{(2) OpenEdition}

\section{Journals}

\section{Édition électronique}

URL : http://journals.openedition.org/artefact/1086

DOI : $10.4000 /$ artefact. 1086

ISSN : 2606-9245

Éditeur :

Association Artefact. Techniques histoire et sciences humaines, Presses universitaires du Midi

\section{Édition imprimée}

Date de publication : 30 mai 2018

Pagination : 65-77

ISBN : 978-2-7535-7494-6

ISSN : 2273-0753

\section{Référence électronique}

Isabelle Rodet-Belarbi, « La transformation des matières dures d'origine animale en Gaule romaine ateliers urbains et artisans itinérants », Artefact [En ligne], 7| 2017, mis en ligne le 29 janvier 2019, consulté le 06 mars 2020. URL : http://journals.openedition.org/artefact/1086 ; DOI : https://doi.org/ 10.4000/artefact.1086 


\section{La transformation des matières dures d'origine animale en Gaule romaine : ateliers urbains et artisans itinérants}

Isabelle RODET-BELARBI *

\section{Résumé}

Les fouilles effectuées à l'emplacement d'anciennes agglomérations gallo-romaines permettent d'enrichir au fur et à mesure l'inventaire des ateliers consacrés au travail de l'os et du bois de cervidé. Ces espaces sont, en effet, rarement répertoriés mais leur place, même discrète dans le paysage urbain, est bien attestée par les très nombreux déchets mis au jour en position secondaire dans les dépotoirs. Par ailleurs, la découverte de rejets de fabrication d'objets dans les villae pose la question de l'existence et du mode de fonctionnement des artisans occasionnels et/ou itinérants travaillant à la demande, de lieu en lieu, voire de ville en ville.

Mots-clés : Bois de cerf, os, Matières dures d'origine animale (MDOA), artisanat, artisan itinérant, ateliers urbains, Gaule romaine.

\section{Abstract. Transformation of hard animal material in the Roman period in Gaul : urban workshops and itinerant artisans}

*. Isabelle Rodet-Belarbi est archéozoologue à l'Inrap, CEPAM-UMR 7246, Nice. Elle étudie la faune des périodes proto- et historiques, et les mobiliers en matière dure d'origine animale des périodes historiques. Parmi ses publications : avec Michiel GAZENBEEK, «L'exploitation des bois de cervidés à l'époque mérovingienne sur le site de Mondelange (Moselle) ", Revue Archéologique de l'Est, t. 63, 2014, p. 259-300 ; avec Gaëtan JouAniN et Benoît ClAvel, « La gestion des restes d'équidés en ville et à la campagne en France au Moyen Âge et au début de l'époque Moderne ", in Élisabeth Lorans (dir.), Le cheval au Moyen Âge, Tours, Presses universitaires François-Rabelais, 2017, p. 345-366. Contact : [isabelle.rodet-belarbi@ inrap.fr]. 
Excavations of ancient Gallo-Roman sites have enriched our knowledge of workshops for making of objects in bone and antler. These are less numerous than by- products found in secondary contexts in dumps, but there is no doubt about their even rare occurrence in the urban landscape. Otherwise, the discovery of secondary products resulting from the manufacture of objects in villae points to the question of the existence and functioning of casual and/or traveling artisans working from orders, moving from one place to the next, maybe even from town to town.

Keywords : Antler, bone, Hard Animal Material (HAM), handcraft, itinerant artisans, urban workshop, Gallo-Roman period.

Le travail de la matière dure d'origine animale durant la période gallo-romaine est fréquemment illustré par la découverte de rejets de matière première (élimination par exemple des extrémités articulaires d'un os long), de déchets de transformation de la matière première via le débitage et le façonnage, d'ébauches et de ratés de fabrication et enfin, d'objets finis. Toutes ces catégories sont diversement représentées, de quelques pièces à plusieurs centaines mises au jour dans des contextes archéologiques variés. La participation des archéozoologues aux études de ce mobilier depuis ces vingtcinq dernières années a permis l'identification précise des matières premières utilisées et l'augmentation de la liste des taxons et des parties anatomiques employés. Quelques objets sont en ivoire, rarement identifiés spécifiquement, mais les déchets et les copeaux de cette matière témoignant de sa transformation en Gaule romaine sont exceptionnels; ils n'ont été observés, à ce jour, que sur deux sites : Lyon (période augustéenne; Rhône ${ }^{1}$ ) et Fréjus (première moitié III siècle; $\left.\operatorname{Var}^{2}\right)$.

Le terme " artisan » désigne ici une personne possédant, d'une part, un savoir-faire indéniable dans le domaine de la transformation de la matière osseuse et, d'autre part, les outils adéquats. Certaines pièces demandent en effet une dextérité indubitable et la différence d'achèvement entre deux objets d'une même catégorie semble relever plus d'une absence de finition, donc d'un temps de travail plus court et d'un coût moindre du produit final, que $\mathrm{du}$ travail d'un " amateur $»^{3}$. Outre ce degré de technicité, au moins un autre critère peut être retenu ${ }^{4}$. Il s'agit de la périodicité de l'activité. La question du statut de ces artisans et celle de leur place dans la société est posée depuis de nombreuses années ${ }^{5}$. Les sources écrites et l'épigraphie ne nous donnent, en effet, aucune information sur ceux qui produisaient des objets en matières osseuses, et l'archéologie nous renseigne plus sur l'activité elle-même que sur ses acteurs. Qu'ils soient établis en milieu urbain ou dans les villae, ces derniers travaillaient-ils l'os et le bois de cervidé « à temps plein » ou transformaient-ils également d'autres matériaux, tels que la corne ou le bois végétal? En milieu rural, certaines personnes étaient-elles employées pour les travaux agricoles et également, compte tenu de leur savoirfaire, à la fabrication de quelques objets ? 
Complémentairement ou parallèlement à ce travail occasionnel effectué par une personne à demeure et perceptible par le biais de déchets ponctuels, peut-on reconnaître et caractériser (en termes de produits et compétences particulières) celui d'artisans itinérants? L'objectif de ce travail est de dresser un bilan de nos connaissances sur ces questions à tra- vers la prise en compte de divers cas de figure et leur mise en perspective, pour identifier des indices qui nous permettront d'aborder le fonctionnement plus ou moins intensif des ateliers urbains et la présence concomitante d'artisans itinérants et/ou occasionnels en ville et en milieu rural.

\section{Les ateliers urbains}

Les ateliers peuvent être repérés dans la trame urbaine et identifiés lorsque leurs sols sont encore couverts de sciure, d'éclats de matière première, d'ébauches... C'est le cas à Reims (rue Maucroix, Marne ${ }^{6}$ ), où quatre espaces liés au travail de l'os ont été mis au jour. Le sol de deux d'entre eux était jonché de milliers de fragments d'os, de copeaux et de sciure. Très compacte, cette couche de poudre d'os avait, par endroits, jusqu'à 4 à $5 \mathrm{~cm}$ d'épaisseur et contenait des supports en cours de façonnage en forme de baguettes facettées. La présence des déchets et des ébauches illustrant plusieurs étapes de la transformation de la matière osseuse, a permis d'identifier une production d'épingles à tête sphérique. Des ratés de fabrication et des objets finis ont également été mis au jour; il est certain que bon nombre d'entre eux ont été élaborés sur place. Le fonctionnement du premier atelier date du milieu du III $^{\mathrm{e}}$ siècle tandis que celui du second débute vers les années 280-290. Le troisième espace a livré également de nombreux rejets de travail, des ébauches et des objets finis. Distant du premier atelier d'une dizaine de mètres, il peut être partie intégrante de celui-ci ou n'être qu'une annexe car leurs occupations sont contemporaines. Enfin, dans le quatrième secteur, ont été découverts les mêmes types de déchets et d'ébauches mais en position secondaire dans le remplissage de fosses, dans des remblais ou des recharges sur des sols. Tous sont datés du quatrième quart $\mathrm{du} \mathrm{III}^{\mathrm{e}}$ siècle au début $\mathrm{du}$ $\mathrm{IV}^{\mathrm{e}}$ siècle. La production d'épingles et d'aiguilles a aussi été identifiée à Autun (Saône-et-Loire), d'après les déchets retrouvés dans le quartier artisanal de la « rue des Pierres » et dans l'atelier de la «maison aux monnaies radiées » (fin $\mathrm{du} \mathrm{III}^{\mathrm{e}}$ siècle ${ }^{7}$ ). Les nombreux éléments mis au jour montrent toutes les étapes de mise en forme depuis l'ébauche en forme de fuseau jusqu'à l'épingle finie. Un atelier localisé près de la voie d'Agrippa qui traverse Amiens (Somme), a livré également de nombreuses diaphyses d'os longs non façonnées, accompagnées d'aiguilles et d'épingles dont certaines sont en cours de fabrication ainsi que diverses baguettes facettées ${ }^{8}$. Cet artisanat est pratiqué du milieu du $\mathrm{II}^{\mathrm{e}}$ siècle jusqu'au milieu du $\mathrm{III}^{\mathrm{e}}$ siècle. Dans ces 
deux villes, seuls les rebuts osseux ont été retrouvés. Aucune trace de sciure, ni de copeaux n'a été relevée lors de la fouille de ces lieux.

À Chartres (Eure-et-Loir), certains des modestes habitats constitués en îlots, abritaient des artisans. L'interprétation des données archéologiques suggère que l'un d'eux travaillait l'os dans une cave d'environ $4 \mathrm{~m}^{2}$ éclairée par un soupirail aménagé dans la partie supérieure de l'un des murs'. Le sol était jonché de déchets qui peuvent être répartis entre quatre catégories. La première regroupe les os longs découpés, essentiellement des radius de bœuf mais la présence d'humérus, de fémurs et de tibias est également à noter. La deuxième est représentée par les grandes esquilles, les éclats de petite et moyenne taille résultant des découpes et des cassures des diaphyses et la troisième, par les petites esquilles qui proviennent de l'enlèvement de matière à l'aide d'outils de type « gouge ». Elles sont mêlées à de la sciure d'os. Enfin, la dernière catégorie rassemble 388 ébauches et/ou objets semi-finis cassés en cours de fabrication qui renvoient à la fabrication de cuillères à cuilleron rond (Fig. 1). En outre, 286 baguettes grossièrement facettées ont été découvertes associées aux éléments précédents, indiquant une production d'épingles et d'aiguilles... À Poitiers (Vienne), les déchets et les ébauches mis au jour dans un atelier/boutique ouvrant sur une rue antique située sous la rue Henri-Oudin correspondent également à diverses étapes de la fabrication de cuillères à cuilleron circulaire, activité qui a eu cours entre le $\mathrm{I}^{\text {er }}$ siècle et le milieu du II $^{\mathrm{e}}$ siècle $^{10}$. Enfin, à Alésia (Côte-d'Or; 110/120-275), l'atelier 58 a livré de nombreux fragments d'os longs retrouvés dans une importante couche de sciure recouvrant l'aire de travail ${ }^{11}$.

Les extrémités de baguettes facettées rejetées après leur séparation d'avec l'objet achevé, collectées dans les ateliers de Compierre (Nièvre $\left.{ }^{11}\right), \mathrm{d}^{\prime}$ Alésia $^{13}$, d'Autun $^{14}$, de Limoges (Haute-Vienne ${ }^{15}$ ) illustrent l'utilisation du tour à archet. Ces petites pièces de 4 à $8 \mathrm{~cm}$ de long possèdent une extrémité pointée, résul-

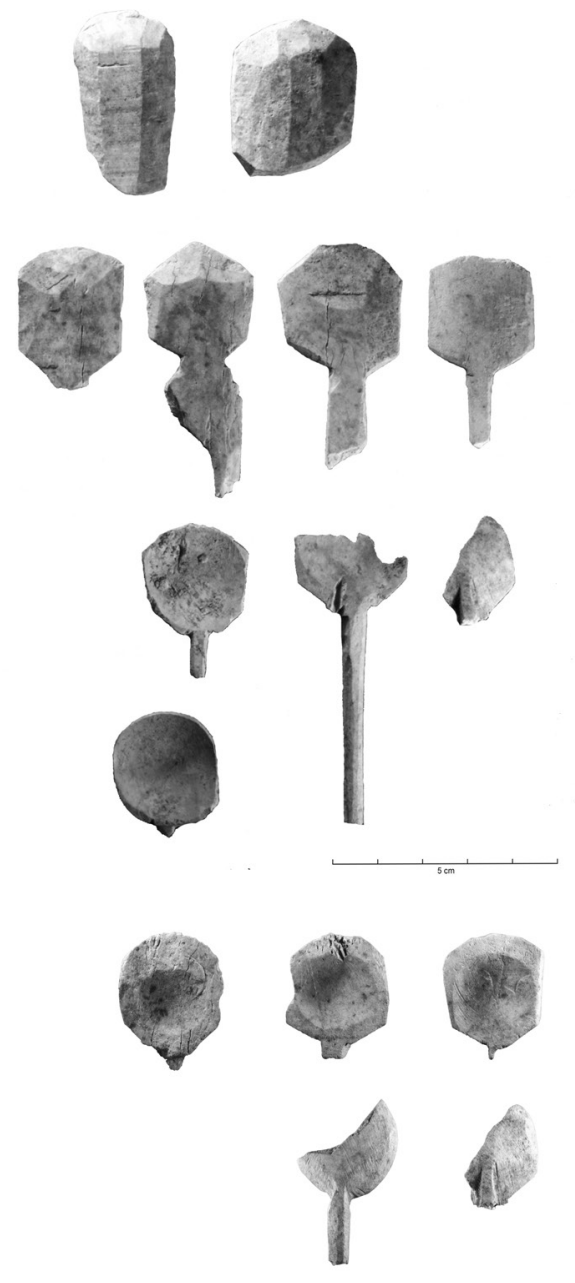

Fig. 1. - Ébauches de cuillères à cuilleron rond découvertes à Chartres. Clichés D. Canny. 

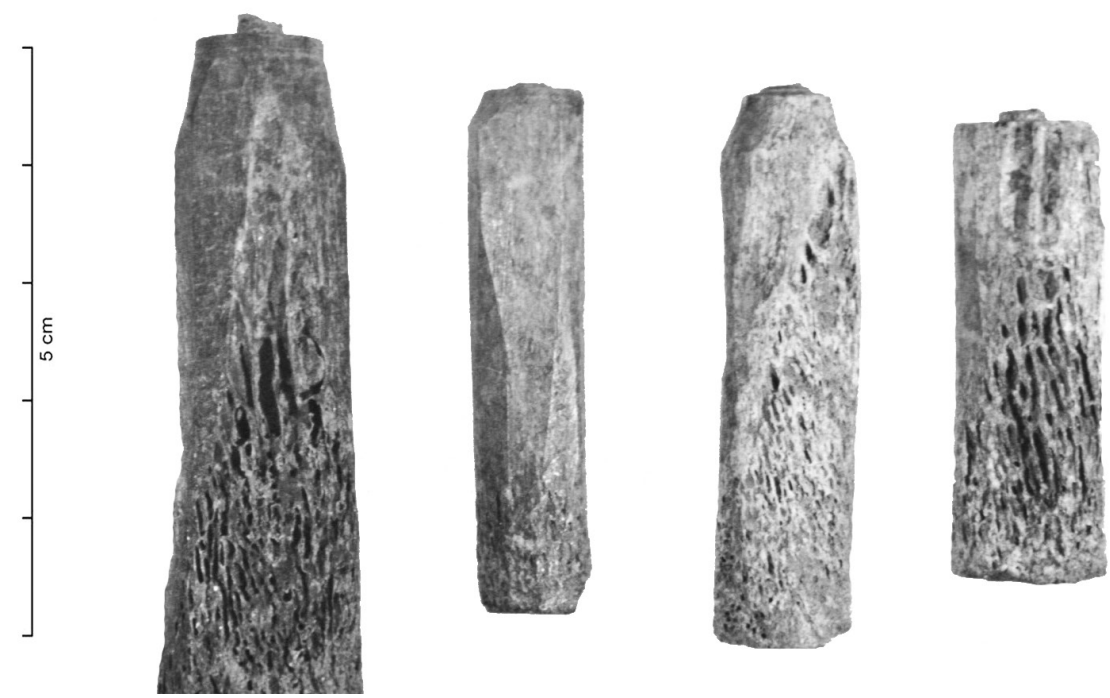

Fig. 2. - Extrémités de baguettes rejetées après la séparation d'avec l'objet tourné découvertes à Autun sur le site du Lycée militaire. Cliché I. Rodet-Belarbi.

tant de leur calage entre les poupées du tour, et une extrémité tournée dont le centre montre la zone d'arrachement d'avec l'objet fini (Fig. 2).

Des corps de métier différents peuvent être associés et partager un même espace. Dans la maison aux Artisans à Autun ${ }^{16}$, il s'agit d'un bâtiment construit en matériaux légers et composé de plusieurs pièces. Les artisans travaillant l'os occupaient quatre d'entre elles tandis qu'un autre espace accueillait, de la fin de la période augustéenne jusqu'au début de la période tibérienne, un cornetier remplacé par des bronziers durant la période flavienne ${ }^{17}$. Une situation similaire peut être signalée pour le site de l'îlot des Cordeliers à Poitiers ${ }^{18}$; le travail de l'os y était associé à celui de métaux (alliages cuivreux, fer et or) de la première $\mathrm{du} \mathrm{II}^{\mathrm{e}}$ siècle au troisième quart du III siècle dans l'un des deux atelierboutiques tandis que dans l'autre, des vestiges artisanaux révèlent le travail $d u$ bronze, du bois de cerf et de l'os. Il en est de même à Saintes (Charente-Maritime) où les artisans travaillant l'os, le fer et/ ou les alliages cuivreux, voire le verre, se partageaient des espaces contigus ${ }^{19}$.

\section{Les dépotoirs urbains}

Les déchets résultant du travail de l'os, mis au jour dans des dépotoirs en divers points des agglomérations, peuvent constituer à eux seuls le remplissage

de ces structures ou être mélangés à d'autres rejets domestiques ou artisanaux. À Alésia par exemple, ils ont été fréquemment mêlés à des résidus liés à 
l'activité des bronziers $^{20}$. Dans tous les cas, leur présence dans des décharges ou des remblais atteste de leur position secondaire. Évacués lors du nettoyage des ateliers, ils correspondent aussi bien aux pièces les plus encombrantes telles que les extrémités proximales et distales des os longs sur lesquels ont été prélevées les diaphyses, réserves de matière première, qu'aux rebuts de taille et aux ébauches abandonnées en cours de travail. Ces déchets en position secondaire ont été découverts dans de nombreuses agglomérations telles que Bavay $\left(\right.$ Nordd $\left.^{21}\right)$, Tours (Indre-et-Loire ${ }^{22}$ ), Lyon $^{23}$, ou encore Javols (Lozère ${ }^{24}$ ) pour ne citer que quelques exemples. Ce sont les seuls indices de cet artisanat; s'ils attestent l'existence d'ateliers dans un environnement certainement proche, ces derniers n'ont néanmoins pas encore été localisés. Au Gué-de-Sciaux (Vienne ${ }^{25}$ ), les indices artisanaux ont été collectés dans des fosses situées dans le sanctuaire. Dans l'agglomération de Fréjus ${ }^{26}$, les rejets de matière première et de travail trouvés ça et là sont à la fois trop peu nombreux et trop disparates pour localiser les atelier(s)-boutique(s) et les objets produits. La maison à l'Ampulla d'Autun située dans la partie occidentale de la ville, non loin du rempart, illustre un cas un peu différent. En effet, plus de 400 extrémités d'os longs et une dizaine de déchets divers ont été mis au jour lors d'un sondage dans un dépotoir, et la localisation d'au moins trois ateliers à l'échelle de cette ville est connue ${ }^{27}$. Toutefois, aucun lien entre les uns et les autres ne peut être établi.

Certains lots de rejets sont composés d'éléments disparates, souvent en faible quantité, empêchant de reconnaître la production des ateliers. En revanche, divers ensembles peuvent renseigner sur l'une ou l'autre des étapes de la préparation de la matière première. À Aixen-Provence (Bouches-du-Rhône), les épandages découverts de part et d'autre de la route antique de Marseille n'ont livré que les extrémités proximales et distales des os des pattes de bovins, leurs diaphyses ayant été récupérées après sciage $^{28}$. En outre, le dépôt occidental contenait également des radius, des tibias et des métapodes d'équidés, eux-aussi sciés. En revanche, les déchets de façonnage et les ratés de fabrication ont été mis au jour essentiellement dans l'espace urbanisé. Même s'ils sont en position secondaire dans les égouts ou les fossés, ils illustrent la fabrication de jetons, d'éléments de charnière et d'aiguilles/ épingles. Ces dépotoirs spécialisés permettent à l'archéologue de proposer une organisation de l'évacuation des déchets, de l'abattage de l'animal aux objets finis. Cette hypothèse repose sur la juxtaposition des rejets résultant, pour les uns, de l'équarrissage des carcasses et, pour les autres, de la préparation de la matière première. Ainsi, les artisans, après avoir récupéré les ossements à travailler, les coupaient dans les ateliers et venaient se débarrasser des parties non exploitées dans les dépotoirs longeant la route, suggérant une affectation préalable des décharges à tel ou tel groupe d'artisans. À Lyon, rue du Chapeau rouge, l'analyse des déchets met en lumière une étape de travail intermédiaire ${ }^{29}$. Les recharges supérieures de la chaussée de la voie ont, en effet, livré des portions de trois types d'os longs sciés et le plus souvent épannelés. Les métapodes sont représentés uniquement par leurs extrémités articu- 
laires, les diaphyses ayant été prélevées pour être façonnées. Les tibias et les fémurs ne sont illustrés que par les portions supérieures et inférieures de leurs diaphyses indiquant que leur partie centrale dont la forme convenait pour les objets envisagés, a été récupérée pour être travaillée. L'absence des surfaces articulaires témoigne de leur élimination préalable au traitement de la diaphyse, et de leur rejet ailleurs. Une fosse découverte à Saintes (Charente-Maritime) contenait de nombreux métapodes de bœuf, dépôt interprété comme une réserve de matière première $^{30}$. À Vertault (Côte-d'Or) ont été recensés, $d$ 'une part, quinze tronçons de diaphyses de tibias et de métapodes de bœuf et d'équidés, débarrassées de leurs extrémités et, d'autre part, 208 plaques taillées longitudinalement dans des diaphyses de radius, de tibia et de métapodes de bœuf et d'équidés, et sciées aux deux extrémités ${ }^{31}$. De nombreuses traces de travail ont été observées à la surface de l'os. Leur destination pourrait être la fabrication d'objets longs et fins (aiguilles, épingles...) et/ou des jetons/ pions.

Certains lots de rebuts de travail très homogènes illustrent les différentes étapes de la chaîne opératoire de l'objet fabriqué. Ainsi, les 248 pièces découvertes dans des remblais à l'ouest d'une des rues du Clos de la Lombarde à Narbonne $^{32}$ (Aude) correspondent à des chutes liées à la fabrication de jetons/ pions (Fig. 3.a) $)^{33}$. Cette même activité a été reconnue à Orange (Vaucluse),
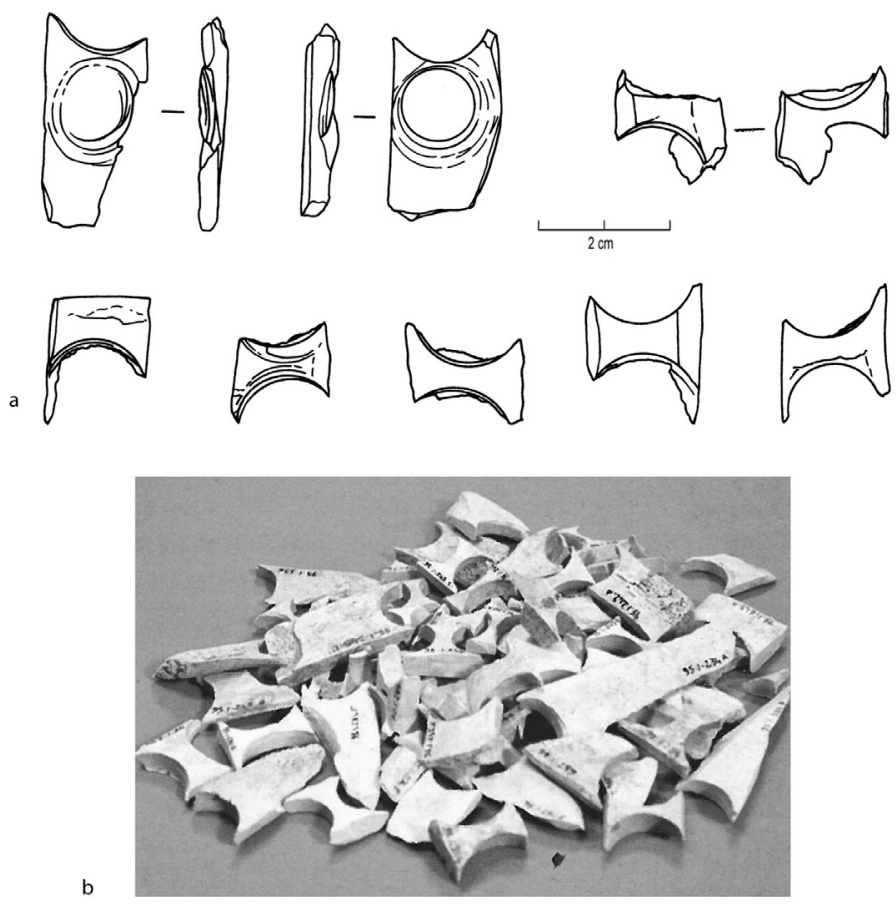

Fig. 3. - Déchets issus de la fabrication de jetons ou de pions. a. - Narbonne, Clos de la Lombarde. Dessins M. Rocheteau. b. - Orange, Saint-Florent. Cliché Ph. Prévot. 
dans le quartier de Saint-Forent, où les plaques osseuses portent le négatif du jeton ou du pion prélevé (Fig. 3.b) ${ }^{34}$. Dans l'agglomération gallo-romaine du VieilÉvreux (Eure), un remblai, comblant en partie un cellier mis au jour dans l'esplanade qui s'est développée près du sanctuaire, contenait de nombreuses pièces qui représentaient « toute la chaine opératoire de la fabrication d'épingles [...], $\mathrm{du}$ plus grossier (os fendus) aux objets finis (épingles polies, complètes, cassées) en passant par les os épannelés, les ébauches d'épingles brisées et les innombrables copeaux d'os agglomérés dans la poudre d' ${ }^{35}{ }^{35} »$. Cette activité, sans doute de très brève durée, aurait fonctionné en 200-220 apr. J.-C. sur l'esplanade du sanctuaire. Des déchets similaires ont été collectés à Amiens (fin I ${ }^{\mathrm{er}}$-milieu III ${ }^{\mathrm{e}}$ siècle ${ }^{36}$ ), au Mans $\left(\right.$ Sarthe $^{37}$ ), à Vieux (fin II ${ }^{\mathrm{e}}$-début III $^{\text {e }}$ siècle; Calvados $^{38}$ ), à Melun (fin I ${ }^{\text {er }}$ début II $^{\mathrm{e}}$ siècle; Seine-et-Marne $\left.{ }^{39}\right)$, à Bordeaux (seconde moitié du III $^{\mathrm{e}}$ siècle; Gironde $^{40}$ ). Par ailleurs, les fouilles sur les sites de Trion et du Verbe incarné à Lyon et de la colline Saint-Eutrope à Orange ${ }^{41}$ ainsi que celles de Saint-Romain-en-Gal (Rhône ${ }^{42}$ ), de Saintes (seconde moitié ${ }^{\text {er }}$ début II $^{\mathrm{e}}$ siècle $^{43}$ ) et du Mans ( $\mathrm{I}^{\mathrm{er}}$ siècle $^{44}$ ) ont livré des rejets et des ébauches d'éléments de charnière.

\section{Les dépotoirs et les ateliers dans les villae}

La découverte d'ateliers ou de leurs dépotoirs en milieu rural est rare. Cette lacune est peut-être en partie due aux choix effectués lors des fouilles qui ont souvent été portés préférentiellement sur la pars urbana. Cependant, quelques exemples peuvent être signalés. Maryline Salin a proposé une synthèse sur les divers déchets mis au jour sur le territoire des Bituriges Cubi ${ }^{45}$. Seules deux mentions sont citées à partir d'éléments ténus à l'exception de la villa des Charnières au Subdray $\left(\mathrm{Cher}^{46}\right)$. La fouille archéologique de cet établissement, éloigné de Bourges d'une dizaine de kilomètres, a permis la découverte d'une fosse de récupération de matériaux utiles à la construction de la villa au cours $\mathrm{du} \mathrm{I}^{\mathrm{er}}$ siècle, reconvertie en dépotoir. Son niveau inférieur contenait de nombreux os sciés; 128 épiphyses et 64 diaphyses plus ou moins épannelées, témoignant de l'exploitation d'ossements d'équidés et de bœuf. Les productions de ce lieu n'ont pas été renseignées par d'autres déchets. Dans son ouvrage sur le Val de Loire, Michel Provost ${ }^{47}$ mentionne la présence d'os sciés découverts près de la galerie de la villa du Dué, évoquant la présence d'un atelier ${ }^{48}$. Le site d'Escolives-Sainte-Camille (Yonne), interprété dans un premier temps comme une agglomération gallo-romaine mais qui correspondrait sans doute plus à une vaste villa ${ }^{49}$, a livré des rebuts de fabrication et des objets partiellement façonnés sur os de bœuf, d'équidé ou de cerf trouvés en divers points ${ }^{50}$. Le lieu de travail avait été identifié dans une cabane construite dans une sorte de cour située à l'arrière de l'aile occidentale de la villa. Cependant, la relecture stratigraphique de tous ces éléments indique une dispersion dans le temps et dans l'espace, 
excluant l'existence d'un atelier ${ }^{51}$. S'il y a bien eu façonnage des os sur le site, leur fabrication, en revanche, semble ponctuelle et pourrait correspondre à des besoins limités qui ont pu être pris en charge par un artisan itinérant ou une personne compétente vivant à demeure. Citons également la villa de Cuperly (Marne) où sept ébauches de règles ont

\section{Synthèse et discussion}

Les quelques études présentées au cours de ce travail rendent comptent des divers cas de figure illustrant l'artisanat de l'os et du bois de cerf et ses différentes facettes telles que l'on peut les percevoir aujourd'hui : déchets en position secondaire et en quantité très variable, grâce auxquels il est possible, ou non, de reconnaître le(s) objet(s) fabriqué(s); sols d'ateliers livrant des rejets de travail, des ébauches et/ou des ratés de fabrication, voire de nombreuses esquilles osseuses et de la sciure. En revanche, les lieux de travail n'ont pas été repérés via l'outillage. Ce dernier, s'il est métallique, est sans doute récupéré tel quel ou refondu ; il a également pu être totalement corrodé et être devenu méconnaissable lors de sa découverte par les archéologues. Par ailleurs, aucun tour ou foret à archet en bois végétal n'a été retrouvé grâce à des conditions exceptionnelles qui auraient permis leur conservation.

Malgré une attention accrue des archéologues sensibilisés à l'existence de cette activité artisanale, et la mise en place de protocoles pour la fouille des ateliers et les études du mobilier osseux permettant une approche précise et une été mises au jour sur le sol de la cave $\mathrm{e}^{52}$. Cette découverte d'objets inachevés liés à l'écriture et à la métrique est un unicum en France et a permis une étude technologique approfondie. Mais, tout comme à Subdray, l'absence d'autres déchets de matière première pose un problème qui ne trouve pas de réponse par l'hypothèse d'un travail ponctuel sur le site.

collecte de données fiables ${ }^{53}$, une dichotomie très nette, en l'état actuel de la recherche, se remarque entre les espaces ruraux et les agglomérations secondaires ou capitales de cité dans lesquelles la répartition spatiale des ateliers est variable ${ }^{54}$; ils sont localisés soit dans le centre, dispersés ou en lien avec d'autres artisanats, soit en périphérie dans des quartiers spécifiques.

Dans les agglomérations, les témoins de l'artisanat de l'os et du bois de cerf sont fréquents, parfois nombreux, qu'il s'agisse des rejets en position secondaire ou de ceux mis au jour sur les sols des ateliers dont les durées de fonctionnement sont datées par le mobilier archéologique associé. Certaines d'entre elles sont longues et l'épaisse couche de sciure découverte sur les sols des ateliers de Reims ou de Chartres par exemple, en témoigne. Elles évoquent en outre une spécialisation vers un type d'objets, afin de rationaliser leur production, peutêtre afin de répondre à des besoins liés à l'urbanisation dès la fin du $\mathrm{I}^{\mathrm{er}}$ siècle. En revanche, que dire des niveaux d'occupation des ateliers qui n'ont livré que quelques ébauches et rejets de fabri- 
cation? Cela suggère-t-il qu'ils étaient plus souvent balayés ou que leur temps d'occupation était plus court? Comment évaluer l'intensité de production d'un atelier ayant fabriqué des épingles ou des éléments de charnières à partir des 340 (Le Mans) ou 400 (Autun) extrémités d'os longs de bœuf? Ou celle de l'atelier d'Orange où il a été retrouvé 66 plaques portant les négatifs des pions/jetons fabriqués, dont la quantité peut être estimée à 103 pièces? S'il est entendu que tous les déchets et les objets façonnés ne peuvent être retrouvés, et que l'état de la recherche ne peut être négligé, il apparait, à ce jour, que les quantités de rejets mis au jour, replacées à l'échelle de l'agglomération, ne constituent pas des masses très importantes ${ }^{55}$. Celles-ci évoquent plutôt des productions ponctuelles destinées à un marché local. Mais il est également possible d'imaginer que le travail de la matière osseuse n'était pas la seule activité des ateliers où ont pu également être transformées d'autres matières dures, animales telle que la corne ou, végétales, telle que le bois. Aucun témoin direct, tels que les déchets de fabrication, n'ont été retrouvés mais

\section{Conclusion}

Ainsi, perçu comme un "bruit de fond ", cet artisanat semble avoir été, dans tous les cas, pratiqué par des « gens du métier ». D'après nos connaissances actuelles, certains d'entre eux ont travaillé en ville, dans des ateliers occupés durant de nombreuses années et ayant produit une grande quantité de pièces, donc une masse importante de rejets, leur absence est liée à leur décomposition dans le sol. En outre, dans le cas du bois végétal, ces déchets ont pu être récupérés comme combustible. Par ailleurs, la présence des artisans itinérants voyageant avec leur savoir-faire et leurs outils est plus souvent imagée pour le milieu rural que pour les zones urbaines. Mais ils ont également pu travailler en ville, sans pour cela être installés de façon pérenne dans un atelier. Et s'il était possible pour eux d'exercer leur métier en milieu rural, les quelques objets en os dont témoignent les rares déchets identifiés pouvaient également être fabriqués par une personne demeurant à la campagne, possédant ce même savoir-faire et œuvrant sur place afin de répondre aux besoins ponctuels ${ }^{56}$. En effet, seuls quelques objets très spécifiques ont peutêtre été produits dans un seul atelier, par exemple les manches de canifs en patte de suidé à Amiens ${ }^{57}$. En revanche, les épingles à tête anthropomorphe stylisée qui montrent des caractéristiques communes ont été façonnées par des " mains » différentes, indiquant au plus la circulation d'un " modèle " réinterprété à chaque nouvelle demande ${ }^{58}$.

d'esquilles et de sciure. Cependant, l'hypothèse $d$ 'ateliers accueillant des artisans polyvalents et habiles dans le travail de la matière osseuse, de la corne et du bois végétal, n'est pas à exclure et permettrait d'expliquer le peu de rejets en os collectés dans certains lieux. La découverte, à ce jour, uniquement de déchets épars en milieu rural permet de poser l'hypo- 
thèse de l'existence concomitante d'artisans itinérants. Mais elle est confrontée à celle qui suppose la présence de personnes ayant ponctuellement la charge de fabriquer des objets dans les villae. Il n'est pas possible, pour le moment, de trancher en faveur de l'une ou de l'autre de ces possibilités.

\section{Remerciements à T. et P. C. Anderson pour cette traduction.}

\section{Notes}

1. Armand Desbat, « L'artisanat antique à Lyon ", in Pascale CHARdron-Picault, Aspects de l'artisanat en milieu urbain : Gaule et Occident romain, Actes du colloque international d'Autun (20-22 septembre 2007), Revue Archéologique de l'Est, 2010, 28 e supplément, p. 71.

2. Isabelle Rodet-Belarbi, Yvon Lemoine, " Objets et déchets de l'artisanat de l'os, du bois de cerf et de l'ivoire à Fréjus (Var) de la période romaine à l'Antiquité tardive ", Revue Archéologique de Narbonnaise, 43, 2010, p. 396.

3 . Il ne nous semble pas recevable que l'artisanat de l'os, du bois de cerf ou de la corne soit effectué par un "bricoleur » comme un passetemps. Ce travail demande une habileté certaine, même effectué ponctuellement.

4. Dans l'introduction du volume de Techniques et culture, intitulé "Spécialisation des tâches et société », divers critères aidant à " la construction d'une typologie des tâches » sont proposés. Ils peuvent tous être retenus dans le cas des ateliers urbains. Sophie Méry, Aline Averbouh, Patrice Brun, Claudine Karlin et Pierre de MiroschedjI «Protocole de comparaison des formes de spécialisation des tâches de d'organisation sociétale ", Techniques et culture, $\mathrm{n}^{\circ}$ 46-47, 2006, p. 7-20.

5. Alain FERDIÈrE, « La "distance critique" : artisans et artisanat dans l'Antiquité romaine et en particulier en Gaule », Les petits cahiers d'Anatole, Laboratoire Archéologie et Territoires, UMR 6575 CNRS université de Tours, $\mathrm{n}^{\circ} 1$, 2001, p. 2-31; Nina CRUMmY, "Bone-working in roman Britain : a model for itinerant craftsmen? ", in Michel POLFER, Artisanat et productions artisanales en milieu rural dans les provinces $d u$ nord-ouest de l'Empire romain, Actes du colloque organisé à Erpeldange (Luxembourg), 4-5 mars 1999 par le Séminaire d'Études Anciennes du Centre universitaire de Luxembourg et Instrumentum, Montagnac, éd. Monique Mergoil, 2001, p. 97-109.

6. Grégory Schütz, Ginette AuxietTe, " Durocortorum rue Maucroix. Un quartier excentré d'une capitale de province romaine (fin du $\mathrm{I}^{\mathrm{er}}$ av. J.-C.-début du IV ${ }^{\mathrm{e}}$ siècle). IV.1. Les ateliers de tabletiers et les objets en os ", Archéologie Urbaine, Bulletin de la Société archéologique champenoise, 104, $\mathrm{n}^{\circ}$ 4, 2011, p. 143-156.

7. Isabelle Rodet-Belarbi, Pascale ChARdronPicAult, « L'os et le bois de cerf à AutunAugustodunum (Saône-et-Loire) : productions et consommation d'un instrumentum ", Revue Archéologique de l'Est, no 54, 2005, p. 149-206.

8. Annick Thuet, « Le travail de l'os dans l'antique Samarobriva (Amiens, F) : première approche ", in Isabelle BERTRAND (dir.), Le travail de l'os, du bois de cerf et de la corne à l'époque romaine: un artisanat en marge?, Actes de la table ronde Instrumentum, Chauvigny (Vienne), 8-9 décembre 2005, Monographies Instrumentum 34, Montagnac, éd. Monique Mergoil, Ass. des Publications de Chauvigny, 2008, p. 35-45.

9. Dominique CANNY, Jean-Hervé Yvinec, «Un atelier de travail de l'os à Chartres au III ${ }^{\mathrm{e}} \mathrm{s}$. apr. J.-C. », in Isabelle BERTRAND (dir.), Le travail de l'os, du bois de cerfet de la corne, op. cit., p. 65-84.

10. Isabelle BERTRAND, « Le travail de l'os et du bois de cerf à Lemonum (Poitiers, F) : lieux de production et objets finis. Un état des données ", in Isabelle BERTRAND (dir.), Le travail de l'os, du bois de cerf et de la corne, op. cit., p. 101-144.

11. Michel MangIN, Un quartier de commerçants et d'artisans d'Alésia. Contribution à l'histoire de l'habitat urbain en Gaule, Bibliothèque Pro Alesia, VIII, université de Dijon, Société des Sciences Historiques et Naturelles de Semur-en-Auxois, 1981, p. 104.

12. Thérèse Poulain-Josien, « Étude de la faune », in Georges FOUET, « La villa galloromaine de Montmaurin (Haute-Garonne) », Gallia, XXe supplément, éd. CNRS, 1969, p. 329.

13. Claude GrAPIN, « L'artisanat de l'os et de la corne ", "Alésia. Comment un oppidum est entré dans 1'Histoire ", Dossiers d'Archéologie, no 305, 2005, p. 116-119.

14. Isabelle Rodet-Belarbi, Pascale Chardron-Picault, " L'os et le bois de cerf... », op. cit.

15. Christian VALLET, « Le travail de la matière animale à l'époque romaine. Le travail de l'os à Augustoritum (Limoges, Haute-Vienne) ", in Isabelle BERTRAND, Actualité de la recherche sur le 
mobilier romain non céramique. Actes du colloque de Chauvigny (Vienne, France), 23-24 oct. 1998, Mémoire XVIII de l'Association des Publications Chauvinoises, 2000, p. 195-214.

16. Isabelle Rodet-Belarbi, Pascale Chardron-Picault, " L'os et le bois de cerf à Autun... »,op. cit., p. 151.

17. Pascale Chardron-Picault, L'artisanat à Autun-Augustodunum : topographie et chronologie des activités manufacturières dans la Civitas Aedorum, thèse de doctorat, université de Bourgogne, Dijon, 2004, 2 vol.

18. Isabelle BERTRAND, « Le travail de l'os et du bois de cerf... », op. cit., p. 109.

19. Karine RobIN, Marilyne SALIN, Isabelle BERTRAND, « Le quartier artisanal de la rue Daubonneau à Saintes (Charente-Maritime) : production de viande et artisanat de l'os aux $\mathrm{I}^{\mathrm{er}}$ et $\mathrm{II}^{\mathrm{e}} \mathrm{s}$. apr. J.-C. ", in Pascale Chardron-Picault, Aspects de l'artisanat en milieu urbain : Gaule et Occident romain, op. cit., p. 407.

20. Claude Grapin, « L'artisanat de l'os et de la corne ", op. cit.

21. Frédéric Loridant, "Pour une approche spatio-temporelle de l'artisanat antique à Bavay », in Jean-Claude BÉAL, Jean-Claude Goyon, Les artisans dans la ville antique, université LumièreLyon 2, Paris, diffusion de Boccard, vol. 6, 2002, p. 121-129.

22. Jean-Philippe Chimier, Didier Dubant, " L'artisanat gallo-romain à Tours (Indre-etLoire) durant le haut-Empire ", in Jean-Claude Beal, Jean-Claude Goyon, Les artisans dans la ville antique, op. cit., p. 173-180.

23. Jean-Claude BÉAL, « Les ateliers galloromains de tabletterie à Lyon et à Vienne ", Latomus, XLII, 3, 1983, p. 607-618.

24. Alain TrintignAC, «État des connaissances sur l'artisanat à Javols/Anderitum, capitale de la cité des Gabales (département de la Lozère), à l'époque gallo-romaine ", in Jean-Claude BEAL, Jean-Claude Goyon, op. cit., p. 221-234.

25. Isabelle BERTRAND, Marilyne SALIN, « Le travail de l'os et du bois de cerf dans un sanctuaire de l'agglomération du Gué-de-Sciaux (Antigny, Vienne) ", in Pascale Chardron-Picault, Aspects de l'artisanat en milieu urbain : Gaule et Occident romain, op. cit., p. 363-381.

26. Isabelle Rodet-Belarbi, Yvon Lemoine, «Objets et déchets de l'artisanat de l'os... », op. cit.

27. Isabelle Rodet-Belarbi, Pascale Chardron-Picault, « L'os et le bois de cerf à Autun...", op. cit.

28. Nùria NiN, « L'artisanat à Aix-en-Provence durant l'antiquité ", in Jean-Claude BEAL, JeanClaude Goyon, Les artisans dans la ville antique, op. cit., p. 235-250.
29. Jean-Pierre LAscoux, Vianney Forest, « Lyon (69) - 9e arr., découverte n 9-4/1 : artisanat de la tabletterie ", in Jean-Claude BÉAL, L'artisanat dans les villes antiques, un bilan, Projet Collectif de Recherches, 1997-1998, p. 70.

30. Karine Robin, Marilyne SALIN, Isabelle BERTRAND, " Le quartier artisanal de la rue Daubonneau... », op. cit.

31. Jean-Claude BÉAL, Isabelle Rodet-Belarbi, « Le travail de l'os, du bois de cerf et de l'ivoire à Vertillum-Vertault (Côte d'Or) », Bulletin Archéologique et Historique du Chatillonnais, $6^{\mathrm{e}}$ série, no 6, 2003, p. 51-104.

32. Les remblais de la rue A sont datés de la seconde moitié du $\mathrm{I}^{\mathrm{er}}$ siècle, de la fin du $\mathrm{II}^{\mathrm{e}}$ - début $\mathrm{III}^{\mathrm{e}}$ siècle et de la fin du $\mathrm{III}^{\mathrm{e}}$-début IV $^{\mathrm{e}}$ siècle.

33. Isabelle Rodet-BELARBI, « Le travail de l'os et l'instrumentum en matière dure d'origine animale ", in Raymond SABRIE, Le Clos de la Lombarde à Narbonne. Atelier de salaisons, thermes, maison IX et rue D, Montagnac, éd. Monique Mergoil, Archéologie et Histoire romaine 29, 2015, p. 299320.

34. Philippe Prevot, «État des connaissances sur la production de l'os à Orange (Vaucluse, F). Étude et comparaison des ateliers du travail de l'os ", in Isabelle BERTRAND (dir.), Le travail de l'os, du bois de cerf et de la corne à l'époque romaine : un artisanat en marge?, op. cit., p. 195-229.

35. Laurent GUYARD, « L'artisanat de l'os dans la ville-sanctuaire gallo-romaine du Vieil-Évreux (Eure). État des connaissances ", in Isabelle BERTRAND (dir.), Le travail de l'os, du bois de cerfet de la corne à l'époque romaine : un artisanat en marge?, op. cit., p. 52.

36. Annick Thuet, « La tabletterie du "Multiplex Gaumont" (ancien "Garage Citroën") à Amiens (Somme) ", Revue Archéologique de Picardie, 2007, n 3-4, p. 1-21; Annick ThuET, « Les matières dures d'origine animale d'époque antique : production et produits finis ", in Éric BINET, "Les fouilles du Palais des Sports/Coliseum à Amiens ", Revue Archéologique de Picardie, 2010, n 27, p. 371-376.

37. Isabelle Rodet-Belarbi, « Le travail de l'os », in Stéphane Deschamps, Michel Vaginay, Le Mans retrouvé. Archéologie, mémoire de la ville, Exposition et catalogue d'exposition, 1990, p. 55.

38. Grégory Sснüтz, « L'artisanat antique dans le chef-lieu de cité de Vieux Aregenua (Calvados) ", in Pascale Chardron-Picault, "Aspects de l'artisanat en milieu urbain : Gaule et Occident romain », op. cit., p. 95-107.

39. Isabelle RODET-BELARBI, «Un atelier du travail de l'os : la fabrication d'épingles » in Sophie Benhaddou, Annie-Claire Lussiez, Jérôme Cottard, Annette Gelinet, Melun au temps de 
Rome, Catalogue d'exposition, musée de Melun, avec le concours scientifique de la DAHIF et la participation du GRAM et de l'ACAME, 1990, p. 46.

40. Pierre CAillat, « La faune des tabletiers de la Cité Judiciaire à Bordeaux », Cité Judiciaire, Bordeaux, DFS de sauvetage urgent du 1/10/2004 au 30/06/1995, ministère de la Culture et de la Francophonie, Service Régional de l'Archéologie Aquitaine, ministère de la Justice DGPPE, AFAN, 1995; Christophe SIREIX, «Bordeaux-Burdigala et la Bretagne romaine : quelques témoins archéologiques du commerce atlantique ", Aquitania, XXI, 2005, p. 241-251.

41. Philippe PrÉvot, «État des connaissances sur la production... », op. cit.

42. Jean-Claude BÉAL, " Les ateliers galloromains... », op. cit.

43. Karine RobIN et al., « Le quartier artisanal de la rue Daubonneau... », op. cit.

44. Isabelle Rodet-Belarbi, « Le travail de l'os » op. cit.

45. Marilyne SALIN, Animaux et territoires. L'apport des données archéozoologiques à l'étude de la cité des Bituriges Cubi ( ${ }^{e r}$ S. av. J.-C., V $V^{e}$ s. apr. J.-C.), Bourges, éd. de Bourges plus, Service d'Archéologie Préventive, Tours, FERACF, 2010.

46. Jean-Philippe Chimier, « La place du monde rural dans la répartition des sites de production d'objets manufacturés. L'exemple des cités des Turons et des Bituriges Cubes », in Michel Polfer, Artisanat et productions artisanales en milieu rural dans les provinces du nord-ouest de l'Empire romain, op. cit., p. 243.

47. Michel Provost, « Le Val de Loire dans l'Antiquité ", Gallia, supp. no 52, éd. CNRS, 1993, p. 255-256; Michel Provost, Carte Archéologique de la Gaule, 41, 1988, p. 172.

48. Ce lot a été découvert lors d'une fouille ancienne et nous n'avons pas eu la possibilité de lire la publication originale.

49. Pascale LAURENT, «Une villa gallo-romaine à Escolives-Sainte-Camille », Bulletin de la Société des sciences historiques et naturelles de l'Yonne, 130 e vol, 1998 , p. $474-479$.

50. Daniel Prost, «Une cabane d'artisans du Iv $^{\mathrm{e}}$ siècle à Escolives-Sainte-Camille », Bulletin de la Société des sciences historiques et naturelles de l'Yonne, 1981, 113, p. 11-51.

51. Isabelle Rodet-Belarbi, Paul Van Ossel, " Les épingles à tête anthropomorphe stylisée ", Gallia, 60, 2003, p. 335.

52. Philippe PrÉvot, « Un atelier de fabrication de règles en os dans une villa gallo-romaine de Cuperly (Marne). Étude technologique d'ébauches de règles réalisées en série ", Revue Archéologique de l'Est, 59, 2010, p. 539-565.
53. Michel Feugère, Vianney Forest, Philippe PRÉVot, «Une grille d'analyse pour décrire et comparer des ateliers de tabletiers? ", in Isabelle BERTRAND (dir.), Le travail de l'os, du bois de cerf et de la corne à l'époque, op. cit., p. 25-33.

54. Martine Joly, « Les artisans dans les capitales de cité de l'est de la Gaule », in Pascale Chardron-Picault, Aspects de l'artisanat en milieu urbain: Gaule et Occident romain, op. cit., p. 33-46.

55. Cette constatation est également faite pour la période médiévale : voir l'article de Dorothée Chaoui-Dérieux et Jean-François Goret.

56. La conclusion de l'étude sur le mobilier en os travaillé de la villa d'Orbe-Boscéaz (canton de Vaud, $\mathrm{CH}$ ) est très intéressante car elle développe cette problématique du travail saisonnier dans une villa même si nous n'adhérons pas à cette image de la personne pour qui " rien de plus simple en effet que de s'asseoir au coin du feu et de tailler, à temps perdu, une diaphyse en forme d'épingle ou d'aiguille à l'aide d'un canif » (voir note 3). Caroline ANDEREs, "La tabletterie », in Daniel Paunier, Thierry Luginbühl, et al., La Villa romaine d'Orbe-Boscéaz, URBA I. La villa romaine d'Orbe-Boscéaz. Genèse et devenir d'un grand domaine rural, Volume 2 : éléments et ornements architecturaux, mobiliers, synthèses, Lausanne, Cahiers d'archéologie romande 162, 2016, p. 405-427.

57. Annick Thuet, « Le travail de l'os dans l'antique Samarobriva (Amiens, F)...», op. cit. p. 43.

58. Isabelle Rodet-Belarbi, Paul VAN Ossel, «Les épingles à tête... », op. cit. 